\title{
Analysis of the improvement in sky coverage for multiconjugate adaptive optics systems obtained using minimum variance split tomography
}

\author{
Lianqi Wang, ${ }^{*}$ Luc Gilles, and Brent Ellerbroek \\ Thirty Meter Telescope Project, 1111 South Arroyo Parkway, Suite 200, Pasadena, California 91105, USA \\ *Corresponding author: lianqiw@tmt.org \\ Received 28 February 2011; revised 18 April 2011; accepted 27 April 2011; \\ posted 29 April 2011 (Doc. ID 143260); published 14 June 2011
}

\begin{abstract}
The scientific utility of laser-guide-star-based multiconjugate adaptive optics systems depends upon high sky coverage. Previously we reported a high-fidelity sky coverage analysis of an ad hoc split tomography control algorithm and a postprocessing simulation technique. In this paper, we present the performance of a newer minimum variance split tomography algorithm, and we show that it brings a median improvement at zenith of $21 \mathrm{~nm}$ rms optical path difference error over the ad hoc split tomography control algorithm for our system, the Narrow Field Infrared Adaptive Optics System for the Thirty Meter Telescope. In order to make the comparison, we also validated our previously developed sky coverage postprocessing software using an integrated simulation of both high-(laser guide star) and low-order (natural guide star) loops. A new term in the noise model is also identified that improves the performance of both algorithms by more properly regularizing the reconstructor. (C) 2011 Optical Society of America

OCIS codes: $\quad 010.1080,010.7350$.
\end{abstract}

\section{Introduction}

Laser guide star (LGS) adaptive optics (AO) systems have been either employed [1-ㅗㄱ or are planned [4, $\underline{5}]$ on most ground-based large-aperture astronomical telescopes to overcome the sky coverage limitations of natural guide star (NGS) AO systems. However, because the laser is projected from the ground and scattered back from the sodium layer, it cannot reliably measure the tip/tilt (TT) wavefront aberration caused by atmospheric turbulence or telescope vibration [6]. The LGS AO systems consequently also needs one or several low-order NGS wavefront sensors (WFS) to provide global and field-dependent TT information, and thus they also have sky coverage limitations. However, the sky coverage limitation is greatly relaxed compared with NGS AO systems, because much less light is required to run these low-

0003-6935/11/183000-11\$15.00/0

(C) 2011 Optical Society of America order NGS WFS at comparatively lower sampling frequencies.

Previously, we reported on high-fidelity sky coverage simulations of multiconjugate adaptive optics (MCAO) systems based on an ad hoc split tomography (AHST) control architecture [7]. There, two TT and three tilt anisoplanatism modes [8] were controlled using a combination of up to one tip/tilt/focus (TTF, $2 \times$ 2 subapertures) and two TT NGS WFS. These five modes are named the NGS modes, because they are controlled by the NGS WFS. A least-squares reconstructor was used to reconstruct these five NGS modes from 12 NGS WFS gradient measurements. This split control enables us to efficiently reconstruct low-order modes in lower frame rates with Type II servo filtering, without introducing low-rank terms to the high-order LGS tomography algorithm [9] that complicate the real-time controller (RTC) and may increase computation latency.

A minimum variance split tomography (MVST) has been reported in $[10,11]$. The MVST control 
architecture is equivalent to integrated tomography when both the LGS and NGS loops are solved exactly (using the Cholesky backsolve for the LGS loop) and run at the same sampling frequency. However, like AHST, MVST enables us to efficiently reconstruct low-order modes in lower frame rates. But unlike AHST, MVST does not make a priori assumptions on the choice of NGS modes and applies corrections to the same number of modes as the number of NGS WFS gradients, instead of only five modes as in AHST. This brings improvement over AHST, as shown by simulation results, without increasing the computation significantly in the RTC. Because MVST does not rely on a priori assumptions on the choice of NGS modes, it can be readily applied to other types of $\mathrm{AO}$ systems, like multiobject $\mathrm{AO}$ (MOAO) or MCAO with more than two deformable mirrors (DMs).

Previous work has assessed the improvement of MVST over AHST in a limited number of simplified cases, that may not represent the performance gain achievable in real-world situations. In this paper, we compare the performance of MVST and AHST in the context of sky coverage, using high fidelity, physical optics simulations, which should closely represent the performance of the real system. We generate 500 Monte Carlo realizations of star fields near the Galactic Pole using the star count statistics model, and we compute the performance of MVST and AHST for each star field. By physical optics, we mean that the Shack-Hartmann WFS sensors are modeled by propagating the wavefront in each subaperture to the far field and sampling the images onto detectors. In this way, realistic model of the Poisson and detector noise can be applied. This is in contrast with geometric optics, where the Shack-Hartmann WFS are simply modeled as averaged gradient or Zernike (best fit) tilt of the wavefront in each subaperture.

To make the comparison, we first validated our previously developed sky coverage postprocessing simulation technique by comparing results with integrated high-/low-order simulations. In the postprocessing simulation technique, the performance of the high-order loop controlled by the LGS WFS is assessed in simulations where ideal corrections on the NGS modes (low order) are applied. In this process, we save the time history of the (i) applied NGS modes ("ideal" values, obtained by fitting the wavefront along the science directions to the corresponding projection of these NGS modes), (ii) NGS WFS point spread functions (PSFs), and (iii) NGS WFS geometric gradient measurements (Zernike tilt). This is valid because to first order, the LGS WFS are insensitive to the NGS modes controlled by the NGS WFS. During the postprocessing step, the performance of the NGS loop controlled by NGS WFS is then assessed for each NGS asterism in the time domain, using the saved time history of the "ideal" NGS modes, and NGS WFS PSF or geometric gradients.

It is important to validate this postprocessing technique because the postprocessing sky coverage simulation is used to select optimal asterisms and corresponding NGS sampling frequencies and Type II control loop gains, for each Monte Carlo realization of star fields, which simulate the stars astronomers will encounter during observations. With the selected optimal asterism for each star field, we compare the performance difference between MVST and AHST using the same control scheme and parameters. Because the postprocessing sky coverage simulation is designed to optimize the performance of AHST, evaluating MVST performance with the selected asterisms and control parameters may be pessimistic, but it at least provides a lower bound of the performance improvement.

We found that MVST generally performs better than AHST, especially in poor conditions where the NGS mode wavefront error is large. MVST shows an improvement of $21 \mathrm{~nm}$ rms over AHST at median sky coverage at zenith.

The paper is organized as follows. Section 2 reviews the mathematical formulation of the MVST method. Section 3 describes the Thirty Meter Telescope (TMT) Narrow Field Infrared Adaptive Optics System (NFIRAOS) that we use for simulations. Section 4 shows the comparison between the postprocessing-b̄ased sky coverage estimates and integrated simulations for AHST. Section 5 shows the performance comparison between A $\mathrm{H}$ ST and MVST. Section $\underline{6}$ presents the conclusions.

\section{Minimum Variance Split Tomography}

The mathematical formulation of MVST has been presented in [11]. We will not repeat the derivation here, but we will show the result and discuss it in the context of sky coverage. The LGS and NGS wavefront reconstruction can be represented as

$$
\begin{gathered}
\hat{x}_{L}=A^{-1} G_{L}^{T} C_{L}^{-1} s_{L}, \\
\hat{x}_{N}=A^{-1} G_{N}^{T} C_{N}^{-1}\left(I+G_{N} A^{-1} G_{N}^{T}\right)^{-1}\left(s_{N}-G_{N} \hat{x}_{L}\right), \\
A^{-1}=\left(G_{L}^{T} C_{n}^{-1} G_{L}+C_{x x}^{-1}\right)^{-1}, \\
\hat{x}=\hat{x}_{L}+\hat{x}_{N},
\end{gathered}
$$

where vectors $s_{L}$ and $s_{N}$ denote the pseudo-open-loop gradient measurements of the LGS and NGS WFS; vectors $\hat{x}_{L}$ and $\hat{x}_{N}$ denote the reconstructed wavefront from LGS and NGS measurements, defined on grids at a few discrete layers where turbulence is significant; matrices $G_{L}$ and $G_{N}$ are the geometric gradient (averaging gradient for LGS and Zernike best fit tilt for NGS) operators for the LGS and NGS from wavefront to WFS subapertures; matrices $C_{L}$ and $C_{N}$ are the covariance matrices of the measurement noise in the LGS and NGS WFSs; $A^{-1}$ (never computed explicitly) is the wavefront reconstructor for the LGS controlled 
modes; and finally $C_{x x}^{-1}$ is the inverse of the covariance matrix of the turbulence, calculated using Ellerbroek's sparse curvature approximation [9].

The tomography is carried out in a two-step process. The high-order reconstruction on LGS WFS measurements is carried out at the rate of the LGS WFS sampling frequency (typically on the order of $1 \mathrm{kHz}$ ) and the low-order reconstruction on the NGS WFS measurements can be carried out at the slower NGS WFS sampling frequency (divisor of the LGS WFS sampling frequency, depending on star brightness and location). When the sampling frequency of the NGS WFS is less than the LGS WFS sampling frequency, the term $G_{N} \hat{x}_{L}$ in Eq. (2) and the DM command used to compute the NGS WFS pseudo-open-loop gradients needs to be averaged over the sampling period of the NGS WFS.

Instead of reconstructing $\hat{x}_{N}$ onto the tomography grid as an intermediate step, we can apply the fitting operator $F$ (see [9] for detailed derivations) in Eq. (2) to reconstruct onto DM actuator commands (denoted as $\hat{a}_{N}$ ) directly to save computations for the RTC:

$$
\begin{gathered}
\hat{a}_{N}=F A^{-1} G_{N}^{T} C_{N}^{-1}\left(I+G_{N} A^{-1} G_{N}^{T}\right)^{-1}\left(s_{N}-G_{N} \hat{x}_{L}\right), \\
F=\left(H_{a}^{T} W H_{a}\right)^{-1}\left(H_{a}^{T} W H_{x}\right),
\end{gathered}
$$

where $H_{a}$ and $H_{x}$ are block matrices of influence functions of optical path differences (OPDs) from the DM actuators and tomography grids to the pupil plane along science directions, and $W$ is the pupil plane amplitude weighting matrix. $W$ can be defined in various ways, depending on the $\mathrm{AO}$ system configuration, but must satisfy the following identity:

$$
\sigma^{2}=\phi^{T} W \phi,
$$

where $\phi$ is the concatenated wavefront error vector defined on the pupil plane along science directions, and $\sigma^{2}$ is the field-averaged rms wavefront error (with piston removed). Reference [9] presents an example of $W$ for an MCAO system.

The first part of this formulation, $F A^{-1} G_{N}^{T} C_{N}^{-1}$, can be viewed as the NGS modes (defined as the modes on the DM controlled by NGS WFS), the middle part, $\left(I+G_{N} A^{-1} G_{N}^{T}\right)^{-1}$, can be viewed as the NGS reconstructor, while the last part, $s_{N}-G_{N} \hat{x}_{L}$, is the conditioning of the NGS WFS measurements to remove the expected value based upon the LGS WFS measurements.

In order to have greater insight into the representation of the NGS modes and to optimize servo gains for each mode, it is useful to orthonormalize the NGS modes. The wavefront variance caused by the NGS modes (denoted as $M_{N}^{\prime} \equiv F A^{-1} G_{N}^{T} C_{N}^{-1}$ ) can be computed as

$$
\sigma^{2}=M_{N}^{\prime T} H_{a}^{T} W H_{a} M_{N}^{\prime},
$$

where $H_{a}$ and $W$ are defined in Eq. (6). Carrying out an eigenvalue decomposition on ${ }_{a}^{T} W H_{a}$ gives
$H_{a}^{T} W H_{a}=U \Sigma U^{T}$, with $U U^{T}=I$. Now we can redefine the NGS modes and NGS reconstructor as

$$
\begin{gathered}
M_{N}=F A^{-1} G_{N}^{T} C_{N}^{-1} U \Sigma^{-1 / 2}, \\
R_{N}=\Sigma^{1 / 2} U^{T}\left(1+G_{N} A^{-1} G_{N}^{T}\right)^{-1}, \\
\hat{a}_{N}=M_{N} R_{N}\left(s_{N}-G_{N} \hat{x}_{L}\right) .
\end{gathered}
$$

The quantity $R_{N}\left(s_{N}-G_{N} \hat{x}_{L}\right)$ is the estimate of the NGS modes in the orthogonalized basis. This estimate is temporally filtered before it is fit to the DMs using $M_{N}$.

\section{TMT NFIRAOS System}

We will compare the performance of minimum variance and AHST in the context of sky coverage for the TMT NFIRAOS. NFIRAOS is an MCAO system with two DMs conjugated to a range of 0 and $11.2 \mathrm{~km}$, respectively, an asterism of six sodium LGSs arranged in a pentagon with a 35 arc sec radius plus one more on axis, and up to three low-order NGS WFSs (one measuring TTF/astigmatism and up to two measuring TT only). The performance requirements for NFIRAOS include diffraction-limited turbulence compensation (with total rms wavefront error not to exceed $187 \mathrm{~nm}$ ) over the field of view (FoV) of up to 30 arc sec in diameter, and high sky coverage (requirement of $2 \mathrm{~m}$ arc sec residual wind shake and turbulence induced TT jitter at 50\% sky coverage near the Galactic Pole). The patrol field for the low-order NGS WFS is consequently a larger, nonvignetted, 2 arc min diameter circular FoV. The NGS WFSs will operate in the near infrared ( $\mathrm{J}$ and $\mathrm{H}$ bands) with $\mathrm{H}$-band Nyquist sampled pixels to maximize linearity. This sets a limiting J-band magnitude of about 22 for sampling frequencies down to $10 \mathrm{~Hz}$.

In both AHST and the proposed MVST, the LGS loop is controlled at $800 \mathrm{~Hz}$, independent of the NGS loop, while the NGS loop will be running at $10-800 \mathrm{~Hz}$, with its output filtered by a Type II controller to have better error rejection at low sampling frequencies $[7,12]$.

Figure 1 shows the LGS and NGS asterism and the DM fitting FoV. Here we have adopted a bigger DM fitting FoV (30" diameter circular) than the science FoV $\left(17^{\prime \prime} \times 17^{\prime \prime}\right.$ square $)$ to improve the NGS sharpening across the 2 arc min diameter without significantly penalizing the performance within the science FoV.

Figure 2 shows a quiver plot of the field distortion effects of each NGS mode in the pupil plane along all evaluation directions for two different asterisms. We can see that the first five modes, which are the global TT and 3 second-order focus and astigmatism-like plate scale modes, are similar to the five modes we control in the AHST (see Eq. 1 in [7]), and they are roughly asterism independent besides a rotation. 


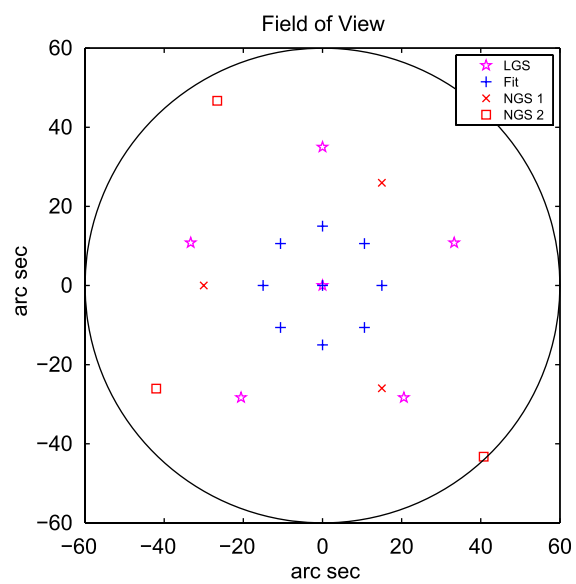

Fig. 1. (Color online) LGS WFS, NGS WFS, and fitting directions in the NFIRAOS FoV for two sample NGS WFS asterisms.

The remaining seven modes, however, are strongly dependent on the NGS asterism.

The sky coverage simulations presented in this paper are carried out at zenith for median seeing conditions at the TMT site, Mauna Kea 13 N. The sevenlayer turbulence profile, derived from DIMM/MASS measurements $[13,14]$, is shown in Table 1 . The Fried parameter is $0.1987 \mathrm{~m}$. The other important

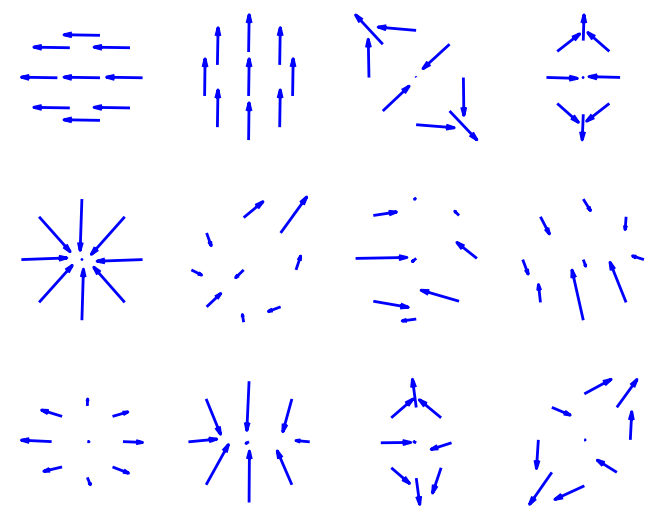

(a)
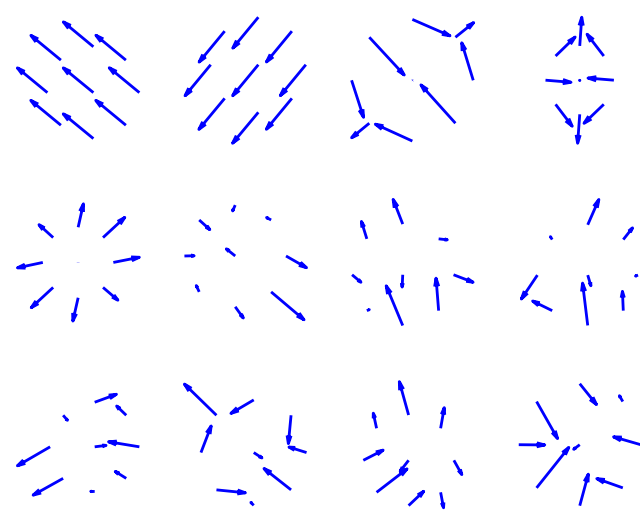

(b)

Fig. 2. (Color online) Field distortion effects of the 12 MVST NGS modes for the two NGS asterisms in Fig. 1 . system and atmospheric parameters are listed in Table $\underline{2}$.

\section{Validation of the Postprocessing Sky Coverage Simulator}

When we first reported the postprocessing sky coverage simulation [7], we only briefly compared with integrated simulations at high sampling frequencies and for a small number of time steps, due to a lack of efficient software and computing resources to do extensive comparisons between the two schemes. It is important to validate the postprocessing sky coverage analysis method because it is used extensively for error budgeting and system parameter trade-offs.

Since the initial development of the code, we have improved this tool by adding an online asterism selection mechanism to remove the dependency on the original geometric sky coverage simulator and to improve the sky coverage by choosing more appropriate asterisms by correctly accounting for the residual errors in NGS controlled modes.

The postprocessing sky coverage simulation is carried out as follows. First, a total of 500 star fields are randomly generated with Galactic Pole guide star statistics [15].

For each star field, we first eliminate stars that do not have diffraction-limited cores (by examining the PSFs). For each star in the field, we then compute the gradient measurement error as a function of NGS WFS sampling frequency (divisors of the LGS sampling rate) based on its brightness and timeaveraged PSF (saved in high-order simulations, as mentioned in Section 1) using the matched-filter [16] algorithm.

We then find all the possible asterisms (combinations of one TTF and a maximum of two TT stars, where the TTF star does not need to be the brightest). For each asterism and at each sampling frequency (we assume that all WFS in the asterism have the same sampling frequency), we can then build the NGS mode reconstructor. The NGS mode reconstructor $G_{M}^{\dagger}$ implements a least-squares estimation of NGS mode $M$ from NGS WFS measurements:

$$
\hat{M}=\arg \min \left\|G_{M} M-s^{N}\right\|_{C_{N}^{-1}}=G_{M}^{\dagger} s_{N}
$$

Table 1. Seven-Layer Turbulence Profile Typical of Mauna Kea $13 \mathrm{~N}^{a}$

\begin{tabular}{cclc}
\hline Layer $i$ & $h_{i}(\mathrm{~m})$ & \multicolumn{1}{c}{$\gamma_{i}$} & $v_{i}(\mathrm{~m} / \mathrm{s})$ \\
\hline 1 & 0 & 0.2887 & 5.6 \\
2 & 500 & 0.17795 & 5.77 \\
3 & 1000 & 0.06602 & 6.25 \\
4 & 2000 & 0.07833 & 7.57 \\
5 & 4000 & 0.1405 & 13.31 \\
6 & 8000 & 0.1216 & 19.06 \\
7 & 16,000 & 0.1269 & 12.14 \\
\hline
\end{tabular}

${ }^{a}$ The height $h_{i}$, relative turbulence strength $\gamma_{i}$, and wind speed $v_{i}$ are shown for each layer $i$. 
Table 2. NFIRAOS and Atmospheric Simulation Parameters

\begin{tabular}{|c|c|}
\hline Parameter & Value \\
\hline Telescope diameter $(D)$ & $30 \mathrm{~m}$ \\
\hline Turbulence outer scale & $30 \mathrm{~m}$ \\
\hline Fried's parameter (median seeing) & $0.1987 \mathrm{~m}$ at $0.5 \mu \mathrm{m}$ \\
\hline $\begin{array}{l}\text { Telescope wind shake ( } 75 \text { th } \\
\text { percentile) }\end{array}$ & 20 mas \\
\hline Mean height of sodium LGS $\left(h_{s}\right)$ & $90 \mathrm{~km}$ \\
\hline DM conjugate altitudes $\left(h_{c}\right)$ & $0,11.2 \mathrm{~km}$ \\
\hline $\mathrm{AO}$ order of correction & $60 \times 60$ \\
\hline $\begin{array}{l}\text { End-to-end optical throughput } \\
\text { for NGS WFS }\end{array}$ & 0.4 \\
\hline Detector passband & $\mathrm{J}$ and $\mathrm{H}(1.25$ and $1.65 \mu \mathrm{m})$ \\
\hline Detector pixel size & $\lambda_{H} /(2 D)$ \\
\hline Detector pixel count & $\begin{array}{c}1024 \times 1024 \text { during } \\
\text { acquisition } \\
\sim 4 \times 4 \text { in closed loop run }\end{array}$ \\
\hline Detector quantum efficiency & 0.8 in both $\mathrm{J}$ and $\mathrm{H}$ bands \\
\hline Detector readout noise & $5 \mathrm{e}^{-} / \mathrm{pixel} / \mathrm{read}$ \\
\hline Detector dark current & $\begin{array}{c}0 \mathrm{e}^{-1} / \mathrm{pixel} / \mathrm{read} \text { at } \\
10-800 \mathrm{~Hz}\end{array}$ \\
\hline Sky background (J band) & 16.25 magnitude/arc $\sec ^{2}$ \\
\hline Sky background (H band) & 14.40 magnitude/arc $\sec ^{2}$ \\
\hline $\begin{array}{l}\text { Intensity of zero-magnitude } \\
\text { star ( } \mathrm{J} \text { band) [18] }\end{array}$ & $3.77 \times 10^{9}$ photons $/ \mathrm{m}^{2} / \mathrm{s}$ \\
\hline $\begin{array}{l}\text { Intensity of zero-magnitude } \\
\text { star (H band) [18] }\end{array}$ & $3.17 \times 10^{9}$ photons $/ \mathrm{m}^{2} / \mathrm{s}$ \\
\hline NGS limiting magnitude & 22 \\
\hline $\begin{array}{l}\text { Number of TTF WFS }(2 \times 2 \\
\text { subapertures })\end{array}$ & 1 \\
\hline $\begin{array}{l}\text { Number of TT WFS (single } \\
\text { subaperture) }\end{array}$ & 2 \\
\hline Science FoV & $17^{\prime \prime} \times 17^{\prime \prime}$ square \\
\hline NGS patrol field FoV & $2^{\prime}$ diameter circular FoV \\
\hline
\end{tabular}

$$
G_{M}^{\dagger}=\left(G_{M}^{T} C_{N}^{-1} G_{M}\right)^{-1} G_{M}^{T} C_{N}^{-1},
$$

where $G_{M}$ is the geometric gradient influence matrix from NGS modes to NGS WFS measurements, $C_{N}$ is the covariance matrix of NGS WFS measurement noise. The estimation error of the wavefront error in NGS modes is then

$$
\sigma_{n}^{2}=\operatorname{Tr}\left(G_{M}^{\dagger} M^{T} H_{a}^{T} W H_{a} M G_{M}^{\dagger} C_{N}\right) .
$$

Let $H_{\text {rej }}$ be the NGS mode servo error rejection function, $H_{n}$ be the noise propagation function, and $\Phi_{N}$ be the sum of the power spectrum density (PSD) of the NGS mode in the atmosphere and telescope wind shake. The calculation of the PSD of the NGS modes caused by turbulence is presented in Appendix A. The total closed loop residual wavefront error in NGS modes can be estimated as

$$
\sigma^{2}=\sigma_{n}^{2} \int \mathrm{d} \nu\left|H_{n}(\nu)\right|^{2}+\int \mathrm{d} \nu\left|H_{\mathrm{rej}}\right|^{2} \Phi_{N}
$$

Here $H_{n}$ and $H_{\text {rej }}$ are functions of the sampling frequency and the control loop gain. We use a Type II controller for NFIRAOS to have better rejection at low frequencies (for details, see Eqs. 16-18 in [7]).
The control loop parameters (Type II loop gain, crossover frequency, and phase lead parameters in our case) can then be optimized for each sampling frequency by minimizing $\sigma^{2}$ while maintaining a phase margin of $45^{\circ}$ and optionally limiting the term $\int \mathrm{d} \nu\left|H_{n}(\nu)\right|^{2} \leq 1$ to not amplify the noise. The optimal sampling frequency for this asterism and the corresponding lowest $\sigma^{2}$ is then recorded.

We then select a few asterisms that have $\sigma^{2}$ near the minimum value in this star field. For each selected asterism, time domain simulations using the previously saved time history of the "ideal" NGS modes and short-exposure NGS WFS PSFs are then carried out, for a few sampling frequencies near the predicted optimal value, to determine the true optimal sampling frequency and final closed-loop wavefront error. The asterism that gives the lowest wavefront error at its optimal sampling frequency will then be selected for this star field, and its performance is recorded for the sky coverage statistics calculation.

In the original prescription, the measurement error of the NGS WFS contained only the gradient measurement error due to photon/detector noise based upon matched filter estimation using time-averaged PSFs. However, we found a significant mismatch between the predicted wavefront error $\sigma^{2}$ and time domain simulation results. We then discovered that the NGS WFS measurement error needed an additional term to capture the aliasing effects caused primarily by the insufficient sensing and correction of the highorder modes by LGS WFS along the NGS directions. Recall that the LGS asterism has 70 arc sec diameter, and FoV of cone beams, while the NGS has a patrol FoV of 2 arc min. The NGS beam passes highturbulence layers that are only partially sensed and corrected by the LGS WFS. The partially corrected NGS wavefront will cause a time-varying measurement error that is stronger when the NGS is further off axis.

To account for this effect, we now have

$$
\begin{gathered}
C_{N}=C_{n}+C_{a}, \\
C_{n}=\left\langle g_{n} g_{n}^{T}\right\rangle, \\
C_{a}=\left\langle g_{a} g_{a}^{T}\right\rangle,
\end{gathered}
$$

where $g_{n}$ is the measurement error due to noise (photon, detector noise), and $g_{a}$ is the gradient error due to the aliasing effect, calculated from the gradients in the stored NGS PSF time history when ideal NGS mode corrections have been applied.

Figure 3 shows the average of the diagonal term in $C_{a}$ for TT NGS WFS at various distances away from the center of the FoV. It can be seen that this effect grows dramatically toward the edge of the FoV to a significant contribution and cannot be neglected. After considering the anisoplanatism effect $C_{a}$ in the NGS measurement error for weighting in the reconstructor $G_{M}^{\dagger}$, we found that the estimated NGS mode wavefront error computed using Eq. (15) is much 
closer to the results obtained in time domain physical optics simulations. Also, we found that "demoting" the TTF WFS to TT WFS (see Subsection 5.F in [7]) is no longer necessary after including $C_{a}$ in $C_{N}$.

It is important to know the temporal PSD of the NGS modes $\Phi_{N}$ in the atmosphere to perform the control loop gain optimization. To achieve this, we have developed a set of formulas that can be used to compute the temporal PSD of any field-dependent or -independent mode in atmospheric turbulence described by Kolmogorov or von Karman statistics. The equations are presented in Appendix A so that the reader can use them to estimate the temporal PSD of the NGS modes, or possibly in other applications.

In addition to developing the above refinements to the sky coverage postprocessing, we have also developed the multithreaded adaptive optics simulator (MAOS), which is a highly efficient, C-based, parallelized simulation code [17] (the source code and documentation are available at this reference). With this software, we complete the performance comparison between the sky coverage postprocessing and integrated simulations in a reasonable amount of time. For each NGS asterism, we run 5000 LGS time steps to let the NGS loop fully settle down and evaluate its performance. With a dual-Xeon W5590-based server, we can run four simulations simultaneously with two threads each. It takes 6.5 wall clock hours to complete all four tasks.

We took the time to run integrated simulations with both LGS and NGS WFSs running in the physical optics mode for each of the 500 asterisms selected by the postprocessing sky coverage code (the asterism in each star field that gives the best performance). The matched filter, sampling frequency, and control loop parameters all took their values from the postprocessing sky coverage code.

Figure 4 shows the performance comparison between the postprocessing sky coverage analysis and integrated simulations, both with the AHST con-

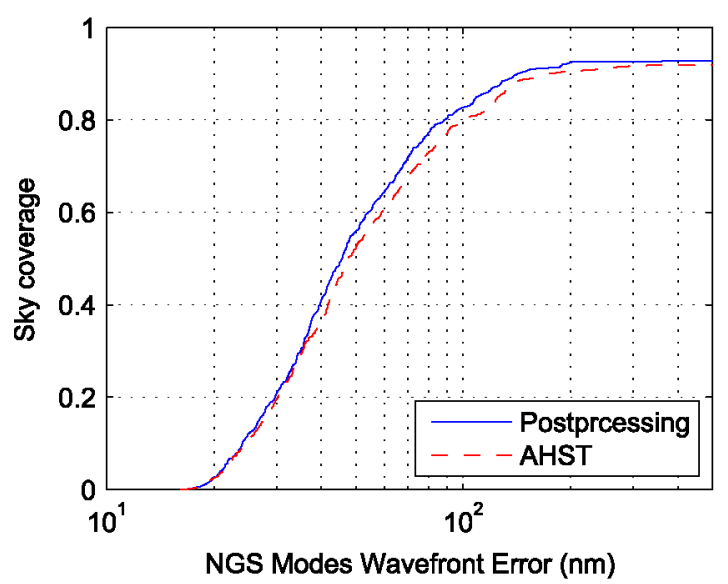

(a)

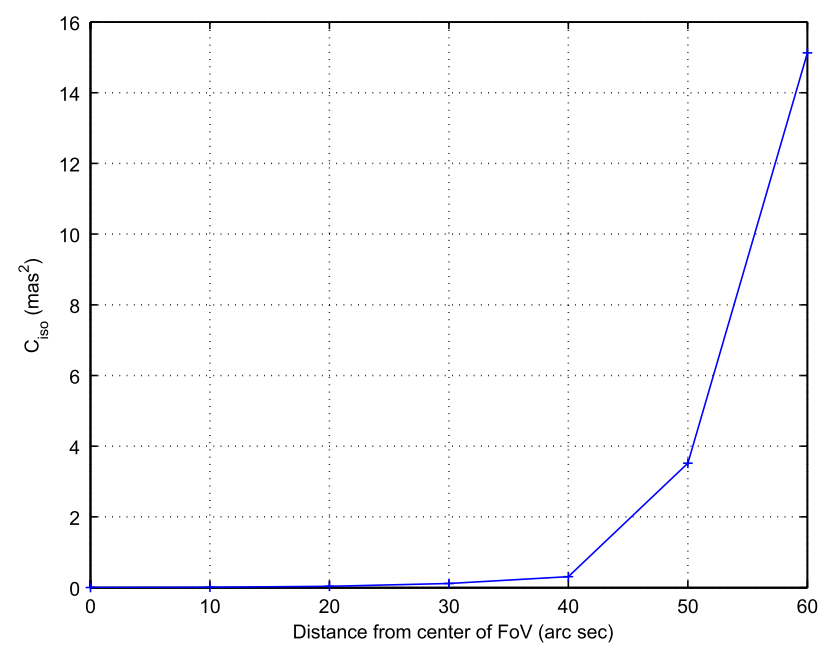

Fig. 3. (Color online) Mean-square NGS WFS measurement error due to the aliasing effect of the higher order atmospheric turbulence left uncorrected by the higher order LGS loop.

trol technique. From the case by case difference plot, we can see that the postprocessing tool tends to underestimate the residual error more when the error in NGS modes is larger, presumably because of the greater impact on LGS WFS dynamic range when the NGS loop performs poorly. The NGS controlled wavefront error at median sky coverage is 46 and $48 \mathrm{~nm}$ in the two cases, with a $13 \mathrm{~nm}$ difference in quadrature. The performance agreement is acceptable given the enormous savings in computing time with the postprocessing sky coverage technique. We allocate the difference of $13 \mathrm{~nm}$ as our algorithm precision term in constructing the NFIRAOS error budget.

\section{Performance Comparison between AHST and MVST}

Because the NGS mode reconstructor for MVST [Eq. (2)] includes a contribution from the LGS tomography output, and the NGS modes now are

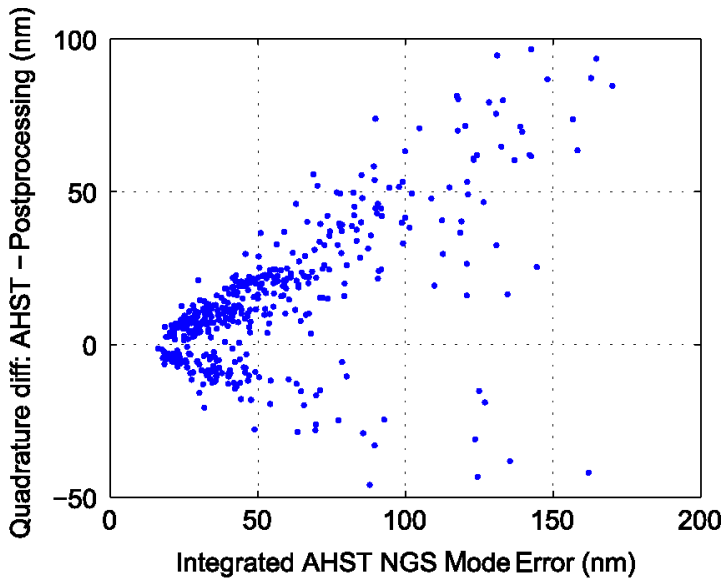

(b)

Fig. 4. (Color online) (a) Comparison between sky coverage results obtained by the postprocessing tool and integrated simulations. (b) From the case-by-case difference plot, we can see that the postprocessing tool tends to underestimate the residual error more when the error is larger, presumably because of greater impact on LGS WFS dynamic range when the NGS loop performs poorly and therefore degrades the sharpening of the NGS further. 
asterism dependent, the postprocessing technique we used for evaluating the sky coverage of AHST cannot be used to evaluate MVST. We instead choose to compare the performance of MVST and AHST using integrated simulations with full physical optics modeling of the LGS and NGS WFS.

We use the same NGS asterism, sampling frequency, and Type II loop gain vectors obtained in postprocessing sky coverage simulations mentioned above for the new MVST simulations. These values may not be optimal for MVST control architecture, but we do not yet have a computationally feasible method to optimize the sampling frequency, control loop gain vectors, and the selection of NGS asterisms.

As mentioned above, MVST controls 12 modes instead of just 5 as in AHST. This introduces a complication in splitting the performance onto THE LGS- and NGS-controlled modes. In AHST, we simply split the performance onto the LGS modes and the five NGS controlled modes. But in MVST, the 12 NGS-controlled modes will be different for each different asterism. We decided to maintain the same split scheme for MVST as in AHST. As a consequence, the seven additional modes controlled by MVST lie in the "LGS," or higher order modes. So we compare the performance difference for these modes, as well as for the "NGS" mode wavefront error, for these two techniques.

Figure 5 shows the performance comparison of the "LGS modes" between AHST and MVST as a function of sky coverage. It remains largely constant for different asterisms in AHST control, albeit with small perturbations due to the high-order effects (for example, when the NGS mode error is large, the high-order measurement will be degraded due to the impacted WFS dynamic range, which consequently impacts the NGS sharpening). But in comparison, it changes significantly for MVST due to the seven additional modes controlled. A median quadratic difference of $5.1 \mathrm{~nm}$ is observed.

Figure 6 shows the performance comparison in the "NGS modes" between the AHST and MVST control architectures in integrated simulations. The MVST has better performance than AHST in a majority

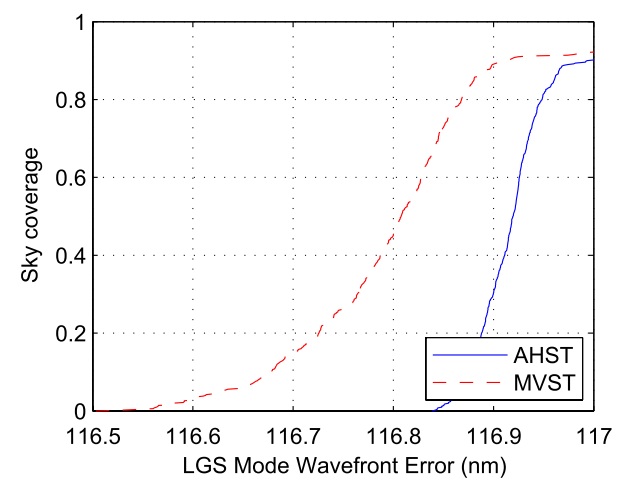

Fig. 5. (Color online) Overall performance comparison of wavefront error in LGS modes between AHST and MVST in integrated simulations. Recall that we have attributed the seven additional modes controlled by MVST to "LGS" modes to have a consistent comparison between the AHST and MVST. The median quadratic difference is $5.1 \mathrm{~nm}$.

of cases, while the improvement tends to grow larger in the poorer cases where the error in the NGS modes is larger. This is particularly beneficial if the telescope has to operate in poor conditions, e.g., in bad seeing, high zenith angle, or with dim stars. The median difference is about $20.4 \mathrm{~nm}$ in quadrature.

In order to get an insight of the improvement in poor conditions, we plotted the time series of the residual wavefront error in the NGS modes for two specific cases (out of the 500 cases studied) in Fig. 7, where the difference between AHST and MVST is large. In case 18, the NGS loop is running at $200 \mathrm{~Hz}$ and closed on physical optics gradients at time step 132. In case 46, the NGS loop is running at $16 \mathrm{~Hz}$ and closed on physical optics gradients at time step 1050 . The performance evaluation is averaged from time steps 2500 to 5000. The improvement of the MVST over AHST in these two cases are 113 and $242 \mathrm{~nm}$ rms in quadrature. It can be seen that the MVST is generally more stable in harsh conditions than is the AHST.

The improvement is visible in all levels of sky coverage, and it is most significant in poor conditions where the wavefront error in the NGS modes is large.

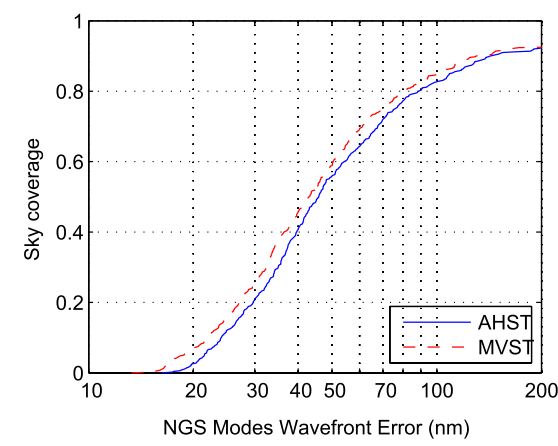

(a)

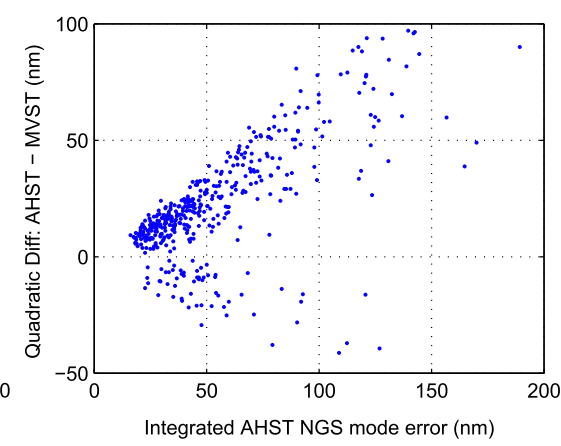

(b)

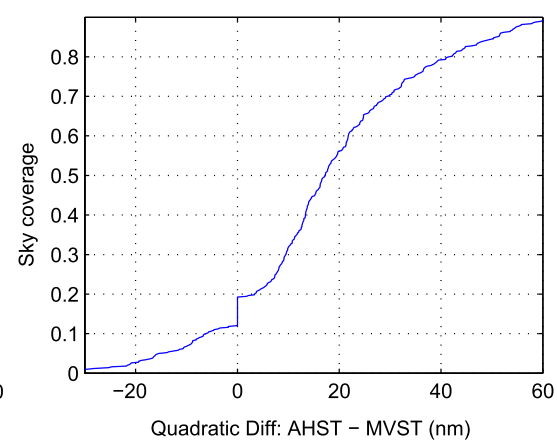

(c)

Fig. 6. (Color online) Comparison between AHST and MVST in integrated simulations: (a) sky coverage, (b) case-by-case difference, and (c) sky coverage of the difference. MVST has better performance than AHST in most of the cases. The improvement tends to grow in those cases where the error in the NGS modes is bigger. The difference at median sky coverage is about $20.4 \mathrm{~nm}$ in quadrature. 


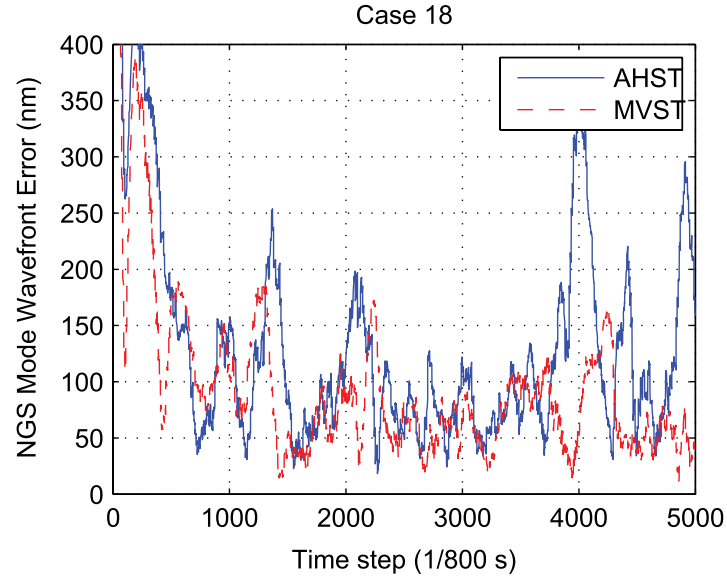

(a)

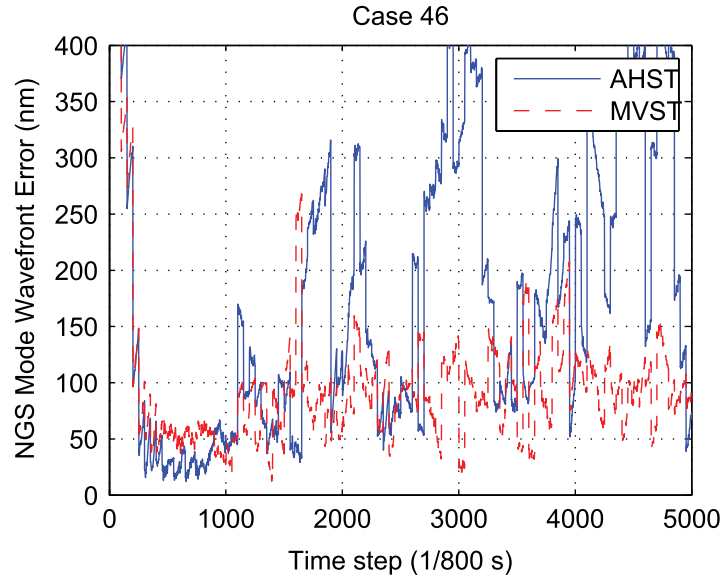

(b)

Fig. 7. (Color online) Comparison between AHST and MVST for two cases where the improvement of MVST over AHST is significant. (a) In case 18, the NGS loop is running at $200 \mathrm{~Hz}$ and closed on physical optics gradients at time step 132. (b) In case 46, the NGS loop is running at $16 \mathrm{~Hz}$ and closed on physical optics gradients at time step 1050 . The performance evaluation is average from time step 2500 to 5000. The improvement of MVST over AHST in these two cases is 113 and $242 \mathrm{~nm}$ in quadrature.

Combining these errors, the net performance improvement provided by the MVST is $21 \mathrm{~nm}$ at zenith at median sky coverage.

\section{Conclusion}

We have described the updates to the postprocessing sky coverage simulation tools with AHST control. We achieve agreement between the results from postprocessing sky coverage simulations and integrated simulations to an accuracy of $13 \mathrm{~nm}$ rms in quadrature.

We have described the formulation of the MVST and compared it against the AHST control architecture. The improvement is visible in all levels of sky coverage, with a median improvement of $21 \mathrm{~nm} \mathrm{rms}$ and is most significant in poor conditions where the wavefront error in the NGS modes is large.

The MVST method can be readily extended to other types of AO systems, because the formulation in Eqs. (1) and (2) only depend on the tomography and do not depend on the science FoV or deformation mirror configuration. The fitting operator in Eq. (6) can be tuned for MOAO, laser tomography AO (LTAO), or other types of AO by redefining $H_{a}$ and $W$ appropriately for each system.

\section{Appendix A: Calculating the Temporal Power Spectrum of Arbitrary Turbulence Modes}

In this section, we describe how to compute the temporal power spectrum of arbitrary modes in the atmosphere that can be field dependent or cross coupled. We considered the case where the wind speed is known and fixed, but the direction of the wind varies uniformly over all possible orientations in the pupil plane.

Define $m_{i}\left(\mathbf{r}, \boldsymbol{\theta}_{j}\right)$ as the projection of mode $i$ along direction $\boldsymbol{\theta}_{j}$, where $\mathbf{r}$ is the spatial coordinate. These modes can be represented as a block matrix of $M$ so that

$$
[M]_{j i}(\mathbf{r})=m_{i}\left(\mathbf{r}, \boldsymbol{\theta}_{j}\right) \sqrt{w_{j}},
$$

where we have multiplied the modes with the scalar weighting of that direction $w_{j}$. Let $\phi_{j}\left(\mathbf{r}, \boldsymbol{\theta}_{j}, t\right)$ be the atmospheric wavefront phase in evaluation direction $\boldsymbol{\theta}_{j}$ at time $t$ and $\phi(\mathbf{r}, t)$ be its block matrix form with weighting so that

$$
[\phi]_{j}(\mathbf{r}, t)=\phi_{j}\left(\mathbf{r}, \boldsymbol{\theta}_{j}, t\right) \sqrt{w_{j}} .
$$

Define the scalar $a(t)_{i}$ as the strength of the mode $i$ contained in the atmosphere at time $t$. We can determine $a(t)$ using least-squares fitting:

$$
a(t)=\arg \min _{a}\|M a-\phi(\mathbf{r}, t)\|_{W}^{2},
$$

where the pupil plane amplitude weighting function $W$ takes the form as defined in Eq. (7).

Following some elementary linear algebra calculations, Eq. (A3) becomes

$$
\begin{aligned}
a(t) & =\left(M^{T} W M\right)^{-1} M^{T} W \phi(\mathbf{r}, t)=C^{-1} b(t) \\
b(t) & =M^{T} W \phi(\mathbf{r}, t) \\
C & =M^{T} W M .
\end{aligned}
$$

The wavefront variance in these modes is then

$$
\sigma^{2}(t)=\|M a\|_{W}^{2}=a^{T}(t) C a(t)=b^{T}(t) C^{-1} b(t) .
$$

We also have

$$
\left\langle a(t) a^{T}(t+\tau)\right\rangle=C^{-1}\left\langle b(t) b^{T}(t+\tau)\right\rangle C^{-1} .
$$

The temporal power spectrum of the wavefront variance is then 


$$
\begin{aligned}
\sigma^{2}(\nu) & =\mathcal{F}\left[\left\langle a^{T}(t) C a(t+\tau)\right\rangle\right] \\
& =\sum_{i i^{\prime}} C_{i i^{\prime}} \mathcal{F}\left[\left\langle a_{i}(t) a_{i^{\prime}}(t+\tau)\right\rangle\right] \\
& =\sum_{i i^{\prime}} C_{i i^{\prime}}^{-1} \mathcal{F}\left[\left\langle b_{i}(t) b_{i^{\prime}}(t+\tau)\right\rangle\right],
\end{aligned}
$$

where $\mathcal{F}$ represent the Fourier transform.

The three-dimensional, continuous atmospheric turbulence is usually approximated by the sum of multiple discrete layers. Therefore, the OPD along direction $k$ at time $t$ can be expressed as

$$
\phi\left(\mathbf{r}, \boldsymbol{\theta}_{k}, t\right)=\sum_{m} \phi_{m}\left(\mathbf{r}+h_{m} \boldsymbol{\theta}_{k}-t \mathbf{v}_{m}, t\right),
$$

where $\phi_{m}, h_{m}$, and $\mathbf{v}_{m}$ are the OPD, altitude, and wind velocity at layer $m$.

Equation (A4) then becomes

$$
\begin{aligned}
b_{i}(t) & =\sum_{k m} w_{k} \int \mathrm{d} \mathbf{r} m_{i k}(\mathbf{r}) W(\mathbf{r}) \phi_{m}\left(\mathbf{r}+h_{m} \boldsymbol{\theta}_{k}-t \mathbf{v}_{m}, t\right) \\
& =\sum_{m} \int \mathrm{d} \mathbf{r}\left[\sum_{k} w_{k} m_{i k}\left(\mathbf{r}-h_{m} \boldsymbol{\theta}_{k}\right) W(\mathbf{r})\right] \phi_{m}\left(r-t \mathbf{v}_{m}, t\right) \\
& =\sum_{m} \int \mathrm{d} \mathbf{r} M_{i m}(\mathbf{r}) \phi_{m}\left(\mathbf{r}-t \mathbf{v}_{m}, t\right) \\
& =\sum_{m} \int \mathrm{d} \boldsymbol{\kappa} \hat{M}_{i m}^{*}(\boldsymbol{\kappa}) \phi_{m}(\boldsymbol{\kappa}) e^{-2 \pi i t \kappa \cdot \mathbf{v}_{m}}
\end{aligned}
$$

where $M_{i m}(\mathbf{r})$ and $\hat{M}_{i m}(\boldsymbol{\kappa})$ are defined as

$$
\begin{aligned}
M_{i m}(\mathbf{r}) & =\sum_{k} w_{k} m_{i k}\left(\mathbf{r}-h_{m} \boldsymbol{\theta}_{k}\right) W(\mathbf{r}) \\
\hat{M}_{i m}(\boldsymbol{\kappa}) & =\mathcal{F}\left[M_{i m}(\mathbf{r})\right]=\sum_{k} w_{k} \hat{m}_{i k}(\boldsymbol{\kappa}) e^{-2 \pi i h_{m} \boldsymbol{\kappa} \cdot \boldsymbol{\theta}_{k}} .
\end{aligned}
$$

Because $M$ is real valued, we have

$$
\hat{M}_{i m}^{*}(\boldsymbol{\kappa})=\mathcal{F}^{-1}\left[M_{i m}(\mathbf{r})\right] .
$$

Consequently,

$$
\begin{aligned}
\left\langle b_{i}(t) b_{i^{\prime}}(t+\tau)\right\rangle & =\sum_{m, m^{\prime}} \int \mathrm{d} \boldsymbol{\kappa} \int \mathrm{d} \boldsymbol{\kappa}^{\prime} \hat{M}_{i m}^{*}(\boldsymbol{\kappa}) \hat{M}_{i^{\prime} m^{\prime}}\left(\boldsymbol{\kappa}^{\prime}\right)\left\langle\phi_{m}(\boldsymbol{\kappa})\right. \\
& \left.\times \phi_{m^{\prime}}^{*}\left(\boldsymbol{\kappa}^{\prime}\right)\right\rangle e^{-2 \pi i\left[t \boldsymbol{k} \cdot \mathbf{v}_{m}-(t+\tau) \boldsymbol{\kappa} \cdot\left(\mathbf{v}_{m^{\prime}}\right)\right]} . \quad(\mathrm{A} 12)
\end{aligned}
$$

All of these two-dimensional integrals with respect to $\mathbf{r}$ or $\boldsymbol{\kappa}$ are performed over the full plane unless specified otherwise. We assume that different turbulent layers have no correlation, therefore

$$
\left\langle\phi_{m}(\boldsymbol{\kappa}) \phi_{m^{\prime}}^{*}\left(\boldsymbol{\kappa}^{\prime}\right)\right\rangle=\Phi_{m}(\boldsymbol{\kappa}) \delta\left(\boldsymbol{\kappa}-\boldsymbol{\kappa}^{\prime}\right) \delta_{m m^{\prime}}
$$

and we obtain

$$
\begin{aligned}
\left\langle b_{i}(t) b_{i^{\prime}}(t+\tau)\right\rangle & =\sum_{m} \int \mathrm{d} \boldsymbol{\kappa} \hat{M}_{i m}^{*}(\boldsymbol{\kappa}) \hat{M}_{i^{\prime} m}(\boldsymbol{\kappa}) \Phi_{m}(\boldsymbol{\kappa}) e^{2 \pi i \tau \kappa \cdot \mathbf{v}_{m}} \\
& =\sum_{m} \int \mathrm{d} \boldsymbol{\kappa} g_{i i^{\prime} m}(\boldsymbol{\kappa}) e^{2 \pi i \tau \kappa \cdot \mathbf{v}_{m}}
\end{aligned}
$$

where

$$
g_{i i^{\prime} m}=\hat{M}_{i m}^{*}(\boldsymbol{\kappa}) \hat{M}_{i^{\prime} m}(\boldsymbol{\kappa}) \Phi_{m}(\boldsymbol{\kappa}) .
$$

The Fourier transform of this correlation function is then

$$
\begin{aligned}
{\left[\Phi_{t}\right](\nu) } & =C^{-1}\left[\Phi_{t}^{\prime}\right](\nu) C^{-1} \\
{\left[\Phi_{t}^{\prime}\right]_{i i^{\prime}}(\nu) } & =\int \mathrm{d} \tau e^{-2 \pi i \tau \nu}\left\langle b_{i}(t) b_{i^{\prime}}(t+\tau)\right\rangle \\
& =\sum_{m} \int \mathrm{d} \boldsymbol{\kappa} g_{i i^{\prime} m}(\boldsymbol{\kappa}) \delta\left(\boldsymbol{\kappa} \cdot \mathbf{v}_{m}-\nu\right) .
\end{aligned}
$$

The wind velocity can be defined as

$$
\mathbf{v}=R(\theta)\left(\begin{array}{l}
v \\
0
\end{array}\right)
$$

where

$$
R(\theta)=\left(\begin{array}{cc}
\cos \theta & -\sin \theta \\
\sin \theta & \cos \theta
\end{array}\right),
$$

is the rotation matrix, and the wind is along direction $\theta$. Similarly $\kappa$ can be expressed as

$$
\kappa=R(\theta)\left(\begin{array}{c}
T \\
S
\end{array}\right) .
$$

We have

$$
d \boldsymbol{\kappa}=d \kappa_{x} d \kappa_{y}=d T d S \quad d \theta d S=\frac{2}{S} d \kappa_{x} d \kappa_{y},
$$

where the 2 comes from the fact that $S$ goes from $-\infty$ to $\infty$ (i.e., each point $\left(\boldsymbol{\kappa}_{x}, \boldsymbol{\kappa}_{y}\right)$ is sampled twice as $\theta$ and $S$ vary), and

$$
\mathbf{v} \cdot \boldsymbol{\kappa}=T v
$$

$$
\kappa^{2}=T^{2}+S^{2}
$$

Averaging over all possible wind direction $\theta$, Eq. (A16) then becomes

$$
\begin{aligned}
{\left[\Phi_{t}^{\prime}\right]_{i i^{\prime}}(\nu) } & =\sum_{m} \int \mathrm{d} T \int \mathrm{d} S \frac{1}{2 \pi} \int_{0}^{2 \pi} \mathrm{d} \theta g_{i i^{\prime} m}(\boldsymbol{\kappa}) \delta\left(T v_{m}-\nu\right) \\
& =\left.\sum_{m} \frac{1}{2 \pi v_{m}} \int \mathrm{d} S \int_{0}^{2 \pi} \mathrm{d} \theta g_{i i^{\prime} m}(\boldsymbol{\kappa})\right|_{T=\frac{\nu}{v_{m}}} \\
& =\sum_{m} \frac{1}{\pi v_{m}} \int_{|\boldsymbol{\kappa}|>\frac{\nu}{v_{m}}} \mathrm{~d} \boldsymbol{\kappa} \frac{g_{i i^{\prime} m}(\boldsymbol{\kappa})}{\sqrt{|\boldsymbol{\kappa}|^{2}-\left(\nu / v_{m}\right)^{2}}} . \quad \text { (A23) }
\end{aligned}
$$

\section{Evaluation in Polar Coordinates}

Noticing that the atmospheric PSD is circularly symmetric, we can choose to do the integral in polar 
coordinates with the transformation

$$
\begin{gathered}
k=\sqrt{k_{x}^{2}+k_{y}^{2}}, \\
\theta=\arctan \left(k_{y} / k_{x}\right), \\
{\left[\Phi_{t}^{\prime}\right]_{i i^{\prime}}(\nu)=\sum_{m} \frac{1}{\pi v_{m}} \int_{0}^{2 \pi} \mathrm{d} \theta \int_{\frac{\nu}{v_{m}}}^{\infty} \mathrm{d} \kappa \frac{k g_{i i^{\prime} m}(\boldsymbol{\kappa})}{\sqrt{k^{2}-\left(\nu / v_{m}\right)^{2}}} .}
\end{gathered}
$$

Because the modes $m$ may not be circularly symmetric, we may need to interpolate $g_{i i^{\prime} m}(\boldsymbol{\kappa})$ onto $k$, $\theta$ during numerical integrations. The denominator has a pole at the lower limit of the integration. We can cope with this complication by breaking the integration into two parts, namely, (i) do the integral from some margin larger than $\nu / v_{m}$ using numerical integration and (ii) do the integral near the pole analytically by approximating the numerator with its center value, and the integration of the denominator is simply

$$
\int_{\nu / v_{m}}^{\nu / v_{m}+\epsilon} \frac{k \mathrm{~d} k}{\sqrt{k^{2}-\left(\nu / v_{m}\right)^{2}}}=\frac{\nu}{v_{m}} \sqrt{\epsilon^{2}-1} .
$$

This gives a smooth curve of the temporal PSD. Step (i) can also be done in logarithmic spacing also by letting $k=\frac{\nu}{v_{m}} e^{x}$.

\section{Verification of the Temporal PSD}

The integration of the temporal PSD should equal to the variance, i.e.,

$$
\int \mathrm{d} \nu\left[\Phi_{t}^{\prime}\right]_{i i^{\prime}}(\nu)=\left\langle b_{i}(t) b_{i^{\prime}}(t)\right\rangle .
$$

Notice that when changing the order of integration, the following two sequences are equivalent

$$
\int \mathrm{d} \nu \int_{|\boldsymbol{\kappa}|>\frac{\nu}{v_{m}}} \mathrm{~d} \boldsymbol{\kappa} \equiv \int \mathrm{d} \boldsymbol{\kappa} \int_{\frac{\nu}{v_{m}}<|\boldsymbol{\kappa}|} \mathrm{d} \nu
$$

Let

$$
k=\sqrt{k_{x}^{2}+k_{y}^{2}} \quad \frac{\nu}{v_{m}}=k \sin \theta .
$$

We have

$$
\begin{gathered}
\frac{1}{\pi v_{m}} \int_{\frac{\nu}{v_{m}}<|\boldsymbol{\kappa}|} \mathrm{d} \nu\left[k_{x}^{2}+k_{y}^{2}-\left(\frac{\nu}{v_{m}}\right)^{2}\right]^{-1 / 2} \\
\quad=\frac{1}{\pi} \int_{-\pi / 2}^{\pi / 2} \mathrm{~d} \theta \frac{1}{k \cos \theta} k \cos \theta=1,
\end{gathered}
$$

so

$$
\int \mathrm{d} \nu\left[\Phi_{t}^{\prime}\right]_{i i^{\prime}}(\nu)=\sum_{m} \int \mathrm{d} \boldsymbol{\kappa} g_{i i^{\prime} m}(\boldsymbol{\kappa})=\left\langle b_{i}(t) b_{i^{\prime}}(t)\right\rangle .
$$

This work is supported by the Thirty Meter Telescope Project. The authors gratefully acknowledge the support of the TMT partner institutions. They are the Association of Canadian Universities for Research in Astronomy, the California Institute of Technology, and the University of California. This work was supported as well by the Gordon and Betty Moore Foundation, the Canada Foundation for Innovation, the Ontario Ministry of Research and Innovation, the National Research Council of Canada (NRC), the Natural Sciences and Engineering Research Council of Canada, the British Columbia Knowledge Development Fund, the Association of Universities for Research in Astronomy (AURA), and the U.S. National Science Foundation (NSF).

\section{References}

1. P. L. Wizinowich, D. Le Mignant,A. H. Bouchez, R. D. Campbell, J. C. Y. Chin, A. R. Contos, M. A. van Dam, S. K. Hartman, E. M. Johansson, R. E. Lafon, H. Lewis, P. J. Stomski, D. M. Summers, C. G. Brown, P. M. Danforth, C. E. Max, and D. M. Pennington, "The W. M. Keck Observatory laser guide star adaptive optics system: overview," Publ. Astron. Soc. Pac. 118, 297-309 (2006).

2. M. Boccas, F. Rigaut, M. Bec, B. Irarrazaval, E. James, A. Ebbers, C. d'Orgeville, K. Grace, G. Arriagada, S. Karewicz, M. Sheehan, J. White, and S. Chan, "Laser guide star upgrade of Altair at Gemini North," Proc. SPIE 6272, 62723L (2006).

3. M. Le Louarn, N. N. Hubin, and R. Arsenault, "Adaptive optics for second-generation VLT instruments," Proc. SPIE 5490, 248-259 (2004).

4. B. Ellerbroek, S. Adkins, D. Andersen, J. Atwood, C. Boyer, P. Byrnes, R. Conan, L. Gilles, G. Herriot, P. Hickson, E. Hileman, D. Joyce, B. Leckie, M. Liang, T. Pfrommer, J.-C. Sinquin, J.-P. Veran, L. Wang, and P. Welle, "Progress toward developing the TMT adaptive optical systems and their components," Proc. SPIE 7015, 70150R (2008).

5. E. Diolaiti, J.-M. Conan, I. Foppiani, M. Lombini, C. Petit, C. Robert, L. Schreiber, P. Ciliegi, E. Marchetti, M. Bellazzini, L. Busoni, S. Esposito, T. Fusco, N. Hubin, F. Quiros-Pacheco, A. Baruffolo, S. D’Odorico, J. Farinato, B. Neichel, R. Ragazzoni, C. Arcidiacono, V. Biliotti, G. Bregoli, G. Cosentino, and G. Innocenti, "A preliminary overview of the multiconjugate adaptive optics module for the E-ELT," Proc. SPIE $\mathbf{7 0 1 5}$ $70150 \mathrm{U}$ (2008).

6. W. Happer, G. J. MacDonald, C. E. Max, and F. J. Dyson, "Atmospheric-turbulence compensation by resonant optical backscattering from the sodium layer in the upper atmosphere," J. Opt. Soc. Am. A 11, 263-276 (1994).

7. L. Wang, B. Ellerbroek, and J. P. Veran, "High fidelity sky coverage analysis via time domain adaptive optics simulations," Appl. Opt. 48, 5076-5087 (2009).

8. B. L. Ellerbroek and F. Rigaut, "Methods for correcting tilt anisoplanatism in laser-guide-star-based multiconjugate adaptive optics," J. Opt. Soc. Am. A 18, 2539-2547 (2001).

9. B. L. Ellerbroek, "Efficient computation of minimum-variance wave-front reconstructors with sparse matrix techniques," J. Opt. Soc. Am. A 19, 1803-1816 (2002).

10. L. Gilles and B. L. Ellerbroek, "Atmospheric tomography with separate minimum variance laser and natural guide star mode control," in First AO4ELLT Conference-Adaptative Optics for Extremely Large Telescopes (EDP Sciences, 2010), paper 07002.

11. L. Gilles, L. Wang, and B. Ellerbroek, "Minimum variance split tomography for laser guide star adaptive optics," Eur. J. Control 17, 327-334 (2011). 
12. J. Véran, C. Irving, A. Beauvillier, and G. Herriot, "Implementation of type-II tip-tilt control in NFIRAOS with woofertweeter and vibration cancellation," Proc. SPIE 7736, 77364I (2010).

13. L. Wang, M. Schöck, G. Chanan, W. Skidmore, R. Blum, E. Bustos, S. Els, R. Riddle, J. Seguel, T. Travouillon, J. Vasquez, D. Walker, and P. Gillett, "High-accuracy differential image motion monitor measurements for the Thirty Meter Telescope site testing program," Appl. Opt. 46, 6460-6468 (2007).

14. M. Schöck, S. Els, R. Riddle, W. Skidmore, T. Travouillon, R. Blum, E. Bustos, G. Chanan, S. G. Djorgovski, P. Gillett, B. Gregory, J. Nelson, A. Otárola, J. Seguel, J. Vasquez, A. Walker, D. Walker, and L. Wang, "Thirty Meter Telescope site testing I: overview,” Publ. Astron. Soc. Pac. 121, 384-395 (2009).

15. Besançon Astronomical Observatory, http://model.obsbesancon.fr/ (2004).

16. L. Gilles and B. Ellerbroek, "Shack-Hartmann wavefront sensing with elongated sodium laser beacons: centroiding versus matched filtering," Appl. Opt. 45, 6568-6576 (2006).

17. Lianqi Wang, "Multithreaded adaptive optics simulator (MAOS)," http://lianqiw.github.com/maos/ (2011).

18. M. S. Bessell and J. M. Brett, "JHKLM photometry—standard systems, passbands, and intrinsic colors," Publ. Astron. Soc. Pac. 100, 1134-1151 (1988). 